\title{
RESEARCH
}

Open Access

\section{Plasma pTau181 predicts cortical brain atrophy in aging and Alzheimer's disease}

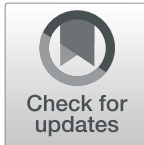

Cécile Tissot ${ }^{1,2,3}$, Andréa L. Benedet ${ }^{1,2}$, Joseph Therriault ${ }^{1,2}$, Tharick A. Pascoal ${ }^{1,2}$, Firoza Z. Lussier ${ }^{1,2}$, Paramita Saha-Chaudhuri ${ }^{4}$, Mira Chamoun ${ }^{1,2}$, Melissa Savard ${ }^{1,2}$, Sulantha S. Mathotaarachchi ${ }^{1,2}$, Gleb Bezgin ${ }^{1,2}$, Yi-Ting Wang ${ }^{1,2}$, Jaime Fernandez Arias ${ }^{1,2}$, Juan Lantero Rodriguez ${ }^{5}$, Anniina Snellman ${ }^{5}$, Nicholas J. Ashton ${ }^{5,6,7,8}$, Thomas K. Karikari ${ }^{5}$, Kaj Blennow ${ }^{5,9}$, Henrik Zetterberg ${ }^{5,9,10,11}$, Etienne De Villers-Sidani ${ }^{12}$, Philippe Huot ${ }^{13}$, Serge Gauthier ${ }^{1,3}$, Pedro Rosa-Neto ${ }^{1,2,3,14,1^{*}}$ (D) and for the Alzheimer's Disease Neuroimaging Initiative

\begin{abstract}
Background: To investigate the association of plasma pTau181, assessed with a new immunoassay, with neurodegeneration of white matter and gray matter cross-sectionally and longitudinally, in aging and Alzheimer's disease.

Methods: Observational data was obtained from the Alzheimer's Disease Neuroimaging Initiative, in which participants underwent plasma assessment and magnetic resonance imaging. Based on their clinical diagnosis, participants were classified as cognitively unimpaired and cognitively impaired. Linear regressions and linear mixedeffect models were used to test the cross-sectional and longitudinal associations between baseline plasma pTau181 and neurodegeneration using voxel-based morphometry.
\end{abstract}

Results: We observed a negative correlation at baseline between plasma pTau181 and gray matter volume in cognitively unimpaired individuals. In cognitively impaired individuals, we observed a negative association between plasma pTau181 and both gray and white matter volume. In longitudinal analyses conducted in the cognitively unimpaired group, plasma pTau181 was negatively correlated with gray matter volume, starting 36 months after baseline assessments. Finally, in cognitively impaired individuals, plasma pTau181 concentrations were negatively correlated with both gray and white matter volume as early as 12 months after baseline, and neurodegeneration increased in an incremental manner until 48 months.

Conclusions: Higher levels of plasma pTau181 correlate with neurodegeneration and predict further brain atrophy in aging and Alzheimer's disease. Plasma pTau181 may be useful in predicting AD-related neurodegeneration, comparable to positron emission tomography or cerebrospinal fluid assessment with high specificity for AD neurodegeneration.

Keywords: Plasma pTau181, Neurodegeneration, Voxel-based morphometry, Alzheimer's disease

\footnotetext{
* Correspondence: pedro.rosa@mcgill.ca

${ }^{1}$ The McGill University Research Centre for Studies in Aging, Douglas

Hospital, McGill University, 875 La Salle Blvd - FBC room 3149, Montreal, QC

H4H 1R3, Canada

${ }^{2}$ Translational Neuroimaging Laboratory-McGill University, Montreal, QC,

Canada

Full list of author information is available at the end of the article
}

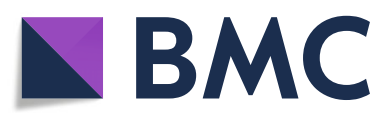

(c) The Author(s). 2021 Open Access This article is licensed under a Creative Commons Attribution 4.0 International License, which permits use, sharing, adaptation, distribution and reproduction in any medium or format, as long as you give appropriate credit to the original author(s) and the source, provide a link to the Creative Commons licence, and indicate if changes were made. The images or other third party material in this article are included in the article's Creative Commons licence, unless indicated otherwise in a credit line to the material. If material is not included in the article's Creative Commons licence and your intended use is not permitted by statutory regulation or exceeds the permitted use, you will need to obtain permission directly from the copyright holder. To view a copy of this licence, visit http://creativecommons.org/licenses/by/4.0/ The Creative Commons Public Domain Dedication waiver (http://creativecommons.org/publicdomain/zero/1.0/) applies to the data made available in this article, unless otherwise stated in a credit line to the data. 


\section{Background}

Advances in quantification of biofluids made possible the detection of Alzheimer's disease (AD) pathophysiological processes in peripheral plasma. It was recently demonstrated that ultra-sensitive tau phosphorylated at threonine-181 (pTau181) in plasma [1-4] provides an inexpensive way to determine the presence of brain neurofibrillary tangles in vivo. Recent studies of plasma pTau181 [1, 5-7] were successful at differentiating AD from other neurodegenerative conditions and presented a strong correlation with pTau181 concentrations in the cerebrospinal fluid (CSF) [1]. Although the associations between CSF and biomarkers of neurodegeneration have been extensively described, little is known regarding plasma pTau181 and its cross-sectional and longitudinal associations with neurodegeneration of white matter (WM) and gray matter (GM). It was however observed that plasma pTau181 levels correlate with lower gray matter volume in the precuneus and temporal lobe of mild cognitive impairment (MCI) and AD participants [6]. Moreover, the novel method to assess plasma pTau181, which is used in the following analyses, has been shown to predict a 1-year cognitive decline and hippocampal atrophy along the AD spectrum [1]. In the current investigation, we examine whether plasma pTau181 correlates with neurodegeneration assessed via voxel-based morphometry (VBM) cross-sectionally, and longitudinally, over a maximum of a 4-year period. We conducted analyses in cognitively unimpaired (CU) and cognitively impaired $(\mathrm{CI})$ individuals, including $\mathrm{MCI}$ and AD participants, who are part of the Alzheimer's Disease Neuroimaging Initiative (ADNI). We hypothesize that plasma pTau181 levels are associated with baseline neurodegeneration of WM and GM, as well as predict subsequent atrophy.

\section{Methods}

\section{Study participants}

Data used in the preparation of this article was obtained from the Alzheimer's Disease Neuroimaging Initiative (ADNI) database (adni.loni.usc.edu). The ADNI was launched in 2003 as a public-private partnership, led by Principal Investigator Michael W. Weiner, MD. The primary goal of ADNI has been to test whether serial magnetic resonance imaging (MRI), positron emission tomography (PET), other biological markers, and clinical and neuropsychological assessment can be combined to measure the progression of mild cognitive impairment (MCI) and early Alzheimer's disease (AD). Data used was downloaded on June 20, 2020. Each ADNI site received approval from their Ethics Board to conduct the study. Written consent was obtained from all the research participants.
The ADNI inclusion/exclusion criteria are described in detail at www.adni-info.org (accessed June 2020). The individuals underwent MRI scans, an assessment of plasma pTau181, and a neuropsychological evaluation. Participants were considered $\mathrm{CU}$ when they had a Clinical Dementia Rating (CDR) of 0 and $\mathrm{MCI}$ when they obtained a CDR of 0.5, while individuals suffering from dementia due to $\mathrm{AD}$ were considered such with a CDR of 1 or higher and met the standard diagnostic criteria for probable AD [8]. The CI group was composed of both MCI and AD individuals. Participants who had no objective evidence of cognitive impairment but reported subjective cognitive decline were analyzed together with the $\mathrm{CU}$ individuals, as per the National Institute of Aging-Alzheimer's Association [9]. Baseline diagnosis was used for statistical analyses.

A detailed description of the sample selection can be found in Fig. 1. The number of individuals at each time point can be found in Table 1 .

\section{Imaging analyses}

Pre-processed 1.5-T and 3-T T1-weighted MRI scans were downloaded from the ADNI database (adni.loni. usc.edu; for pre-processing details, see [10]). Anatomical images were segmented into probabilistic gray matter (GM) and white matter (WM) maps using the SPM12 segmentation tool. Each GM and WM probability map was then non-linearly registered (with modulation) to the ADNI template using DARTEL [11] and smoothed with a Gaussian kernel of full width half maximum (FWHM) of $8 \mathrm{~mm}$. All images were visually inspected to ensure proper alignment to the ADNI template.

\section{Plasma measurements}

Plasma pTau181 was measured using a clinically validated in-house assay described previously [1]. Plasma pTau181 was measured on Simoa HD-X instruments (Quanterix, Billerica, MA, USA) in April 2020 at the Clinical Neurochemistry Laboratory, University of Gothenburg, Mölndal, Sweden, by scientists blinded to participants' clinical information. Plasma pTau181 data was collected over 47 analytical runs. Assay precision was assessed by measuring three different quality control samples at the start and end of each run, resulting in within-run and between-run coefficients of variation of $3.3-11.6 \%$ and $6.4-12.7 \%$ respectively. Out of 3762 ADNI samples, four were removed due to inadequate volumes. The remaining 3758 all measured above the assay's lower limit of detection $(0.25 \mathrm{pg} / \mathrm{ml})$, with only six below the lower limit of quantification $(1.0 \mathrm{pg} / \mathrm{ml})$, which were excluded from the study. 


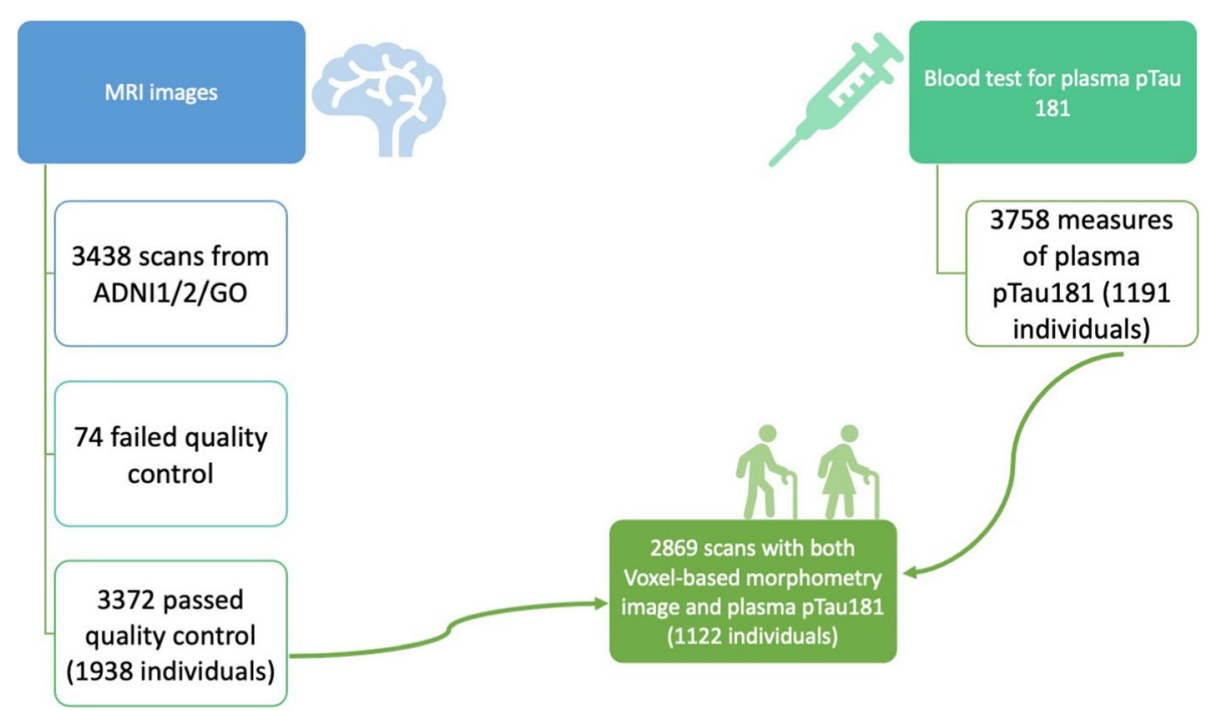

Fig. 1 Sample selection from ADNI. 3438 MRI were available from participants, 74 of them failed quality control, ending up to 3364 available scans to study. Similarly, 3758 participants had available results from plasma ptau181. Combined together, we had 2869 available scans and plasma pTau181 results which dates of assessment were within 6 months

\section{Statistical analyses}

R statistical software package (version 4.0.0) was used to perform the nonimaging statistical analyses. Plasma pTau181 results were log-transformed to meet the requirements of parametric statistics. We first conducted $t$-test (continuous variables) and chi-square tests (categorical variables) for demographics.

We also conducted linear mixed-effect (LME) regression models, using the lme4 package, in order to compare the progression of plasma pTau181 in each diagnostic group over time. The LME included plasma pTau181 as the dependent variable and the interaction between time and group as the independent variable. The covariates were sex and age at baseline. To accommodate the correlation arising from multiple measurements on the same participant, we also included a random intercept. The $95 \%$ confidence intervals were estimated based on the estimated fitted value across the distribution from 1000 simulations of the model that includes all variations. All tests mentioned previously were two-sided with a statistical significance level of $P<.05$.

For brain imaging, we conducted linear models (LM) and LME at the voxel level using VoxelStats [12]. We

Table 1 Number of individuals at each time point

\begin{tabular}{ll}
\hline Time points & Number of participants \\
\hline $\mathrm{BL}$ & $1122(384 \mathrm{CU}$ and $738 \mathrm{Cl})$ \\
V12 & $786(238 \mathrm{CU}$ and $548 \mathrm{Cl})$ \\
V24 & $605(240 \mathrm{CU}$ and $365 \mathrm{Cl})$ \\
V36 & $177(51 \mathrm{CU}$ and $126 \mathrm{Cl})$ \\
V48 & $179(73 \mathrm{CU}$ and $106 \mathrm{Cl})$ \\
\hline
\end{tabular}

studied the associations between log-transformed plasma pTau181 and GM and WM images. Firstly, we investigated the relationship between log-transformed plasma pTau181 and VBM images cross-sectionally for each diagnostic group (CU and $\mathrm{CI}$ ) separately, adjusting for sex and age at baseline. Additionally, we investigated the longitudinal associations between baseline plasma pTau181 and VBM images. The LME included VBM images as the dependent variable and the interaction between time and baseline plasma pTau181 as the independent variable. They were adjusted for sex and age at baseline, as well as random intercept. The model was performed in CU and CI individuals separately. Finally, to infer disease progression at each visit, we used the same statistical model using the follow-up visit (time) as a categorical variable. We used random field theory [13] (RFT) to correct all imaging results for multiple

Table 2 Characteristics of participants included in the study. The sample was composed of 384 cognitively unimpaired individuals, and 738 cognitively impaired, among which 539 were diagnosed with mild cognitive impairment and 199 with probable Alzheimer's disease

\begin{tabular}{lll}
\hline Characteristics & CU & Cl \\
\hline Number of subjects & 384 & 738 \\
Age (mean, SD) in years & $74.40(6.50)$ & $73.61(7.94)$ \\
Females $(n, \%)$ & $205(53 \%)^{\ddagger}$ & $311(42 \%)^{\ddagger}$ \\
MMSE score (mean, SD) & $29.06(1.23)^{\dagger}$ & $26.36(3.68)^{\dagger}$ \\
Plasma pTau181 (mean, SD) & $15.48(10.02)^{\dagger}$ & $19.80(10.75)^{\dagger}$ \\
\hline
\end{tabular}

${ }^{\mp}$ Statistical difference between groups $(P<0.05)$

${ }^{+}$Statistical difference between groups $(P<0.001)$ 
comparisons. Exploratory analyses were also conducted correcting for random slopes.

\section{Results}

\section{Demographics}

Demographic information can be found in Table 2. The sample included a total of 1122 individuals, among which 384 were $\mathrm{CU}$ and 738 were CI $(539 \mathrm{MCI}$ and 199 AD). The average follow-up time was $22 \pm 11.63$ months. There was no statistically significant difference between the groups in terms of age. However, there were statistically significant differences in terms of sex, MMSE scores, and the plasma pTau181 levels between the CU and CI individuals. The CI group was composed predominantly of males and showed lower MMSE scores and higher plasma pTau181 levels. For the CU group, the mean of plasma pTau181 levels was $15.48 \pm 10.02$ $\mathrm{pg} / \mathrm{mL}$, while for the CI, it was $19.80 \pm 10.75 \mathrm{pg} / \mathrm{mL}$ (Fig. 2a).

\section{Regional association between plasma pTau181 with WM and $\mathrm{GM}$ volume predominate in $\mathrm{Cl}$}

Cross-sectional analysis in the $\mathrm{CU}$ group revealed that plasma pTau181 was not associated with WM volume. Nevertheless, there was a negative relationship with GM volume. This association was found in the anterior cingulate and the lateral occipital gyrus (Fig. 2b).
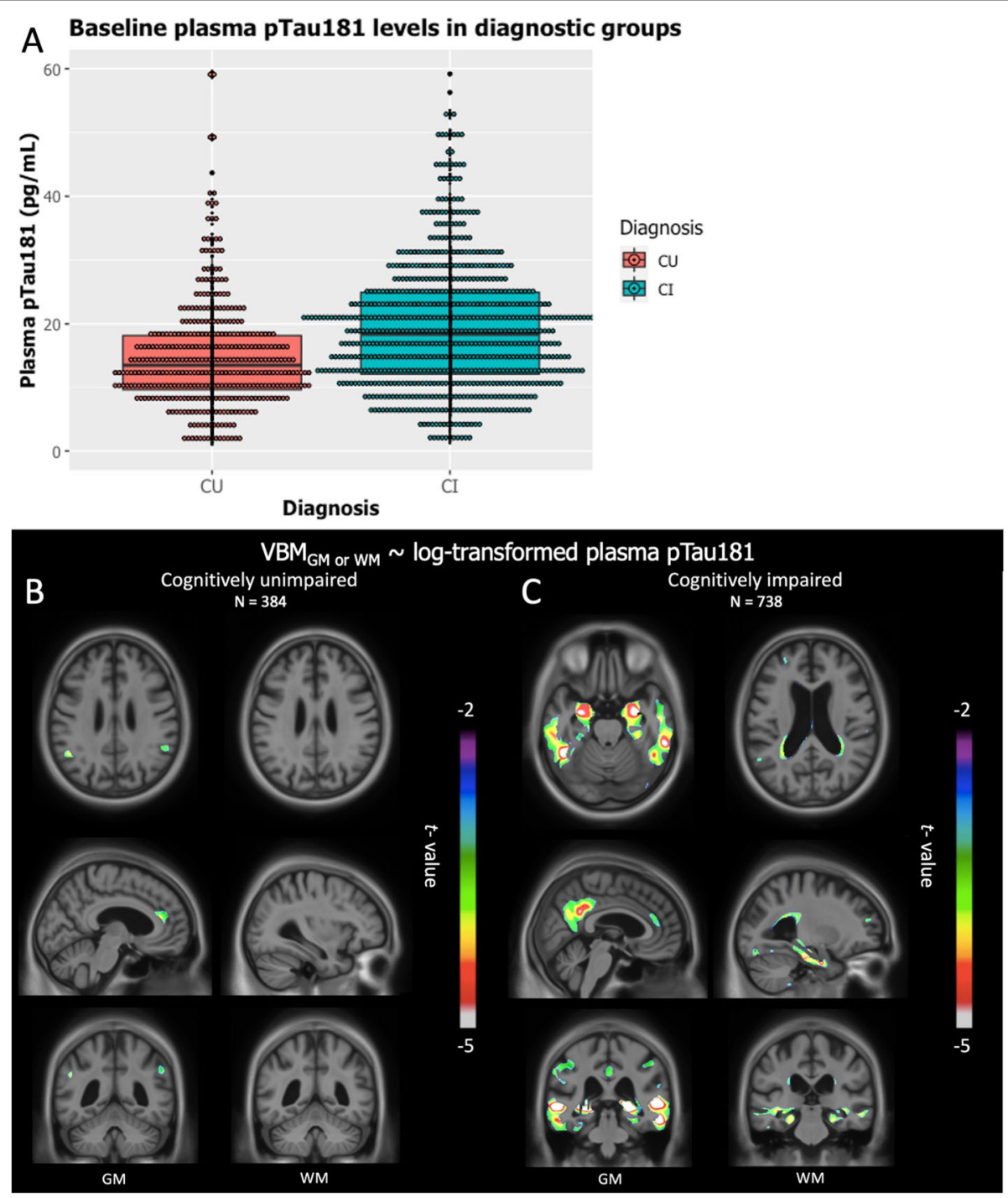

Fig. 2 Associations between plasma tau 181 and neurodegeneration predominate in Cl individuals. a Mean of log-transformed plasma pTau181 depending on the diagnostic group. $\mathbf{b}$ CU individuals showed a negative correlation with GM in the anterior cingulate and occipital gyrus. However, no correlation was found with WM. c Cl individuals presented a negative correlation with GM in the precuneus, anterior cingulate, medial, and lateral temporal gyrus. WM decrease was found in the corpus callosum and the temporal lobe 
In CI individuals, we discovered strong negative associations between plasma pTau181 and GM as well as WM volumes. Correlations between the blood-based biomarker and GM were observed in the precuneus, the anterior cingulate, and the medial and lateral temporal gyrus. WM and plasma pTau181 associations were found in the corpus callosum and the temporal lobe (Fig. 2c). R-maps of the cross-sectional analyses can be found in Supplementary Figure 1.

\section{Plasma pTau181 predicts subsequent GM and WM volume decline}

There was no significant difference in the rate of change of plasma pTau181 between CU and CI groups over a period of 48 months $(P=0.16) \quad$ (Fig. 3a). Individual changes can be seen in Supplementary Figure 2. LME models conducted in the CU group showed a negative association between plasma pTau181 and GM volume in the temporal lobe, the precuneus, and the anterior cingulate cortex. Similar analyses with WM volume changes did not survive correction for multiple comparisons (Fig. 3b). In the CI individuals, negative associations between plasma pTau181 and GM volume were observed in the precuneus and the frontal cortex, with even stronger associations in the temporal area. Plasma pTau181 and WM volume showed a negative association in the corpus callosum and the frontal and temporal lobes (Fig. 3c). When correcting for random slopes, the results were identical.
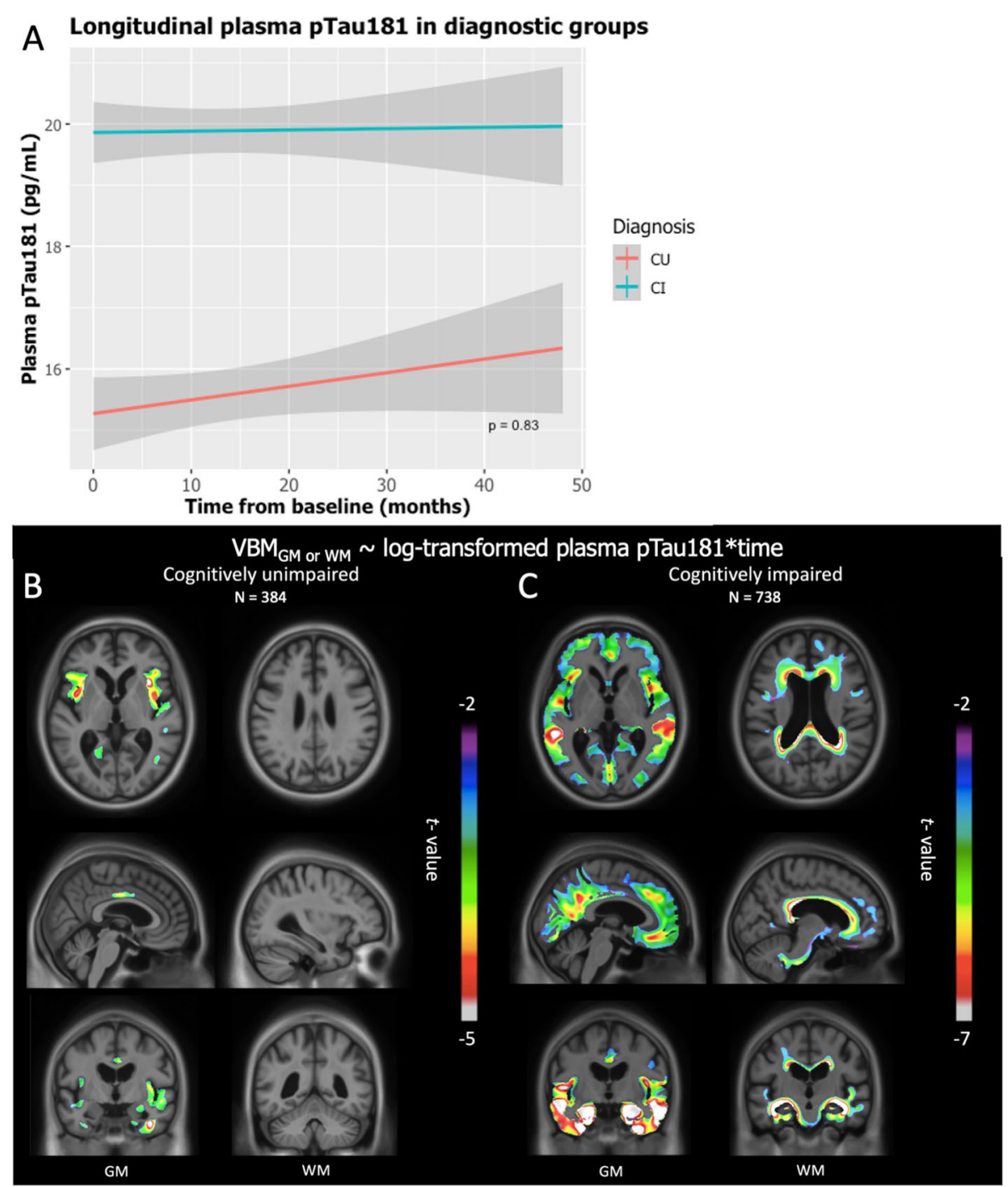

Fig. 3 Association between plasma pTau181 and rate of brain atrophy in AD-related areas. a There is no significant difference between the rates of change of plasma pTau181 between CU and Cl individuals. b CU individuals presented a negative correlation between plasma pTau181 and GM in the temporal lobe, precuneus, and anterior cingulate cortex. No correlation was found with WM. $\mathbf{c} \mathrm{Cl}$ individuals showed a negative correlation with GM in the precuneus, frontal cortex, and temporal lobe. WM results showed a negative correlation in the corpus callosum, the temporal, and the frontal lobes 
Negative associations between plasma pTau181 and GM or WM volume spread over time

Among the $\mathrm{CU}$ individuals, negative correlations between plasma pTau181 with GM were observed at the 36-month FU (Fig. 4a) and the 48-month FU (Fig. 4b) in the precuneus, insula, medial frontal, anterior cingulate, and finally temporal lobe. Comparatively, there was no significant negative relationship with WM volume.

In the CI group, areas in which GM volume negatively correlated with plasma pTau181 progressively expanded from 12- to 48-month FU. As early as 12-month FU, pTau181 and GM volume correlations were restricted to the medial frontal, precuneus, posterior cingulate, and temporal lobe (Fig. 4c). At 24-month FU, these negative correlations also included the medial occipital cortex (Fig. 4d). From 36- (Fig. 4e) to 48-month FU (Fig. 4f), these associations embraced the whole medial frontal cortex, the precuneus, the medial occipital, and the temporal lobes. Plasma pTau181 and WM volume associations similarly progressed over time; these correlations were initially confined to the vicinity of the choroidal fissure as well as the temporal horn of the lateral ventricle at 12-month FU (Fig. 4g). Subsequently, they encompassed the lateral periventricular WM at 24month FU (Fig. 4h), including frontal and occipital lobes at 36-month FU (Fig. 4i) to finally embrace the whole WM 48 months after baseline (Fig. 4j).

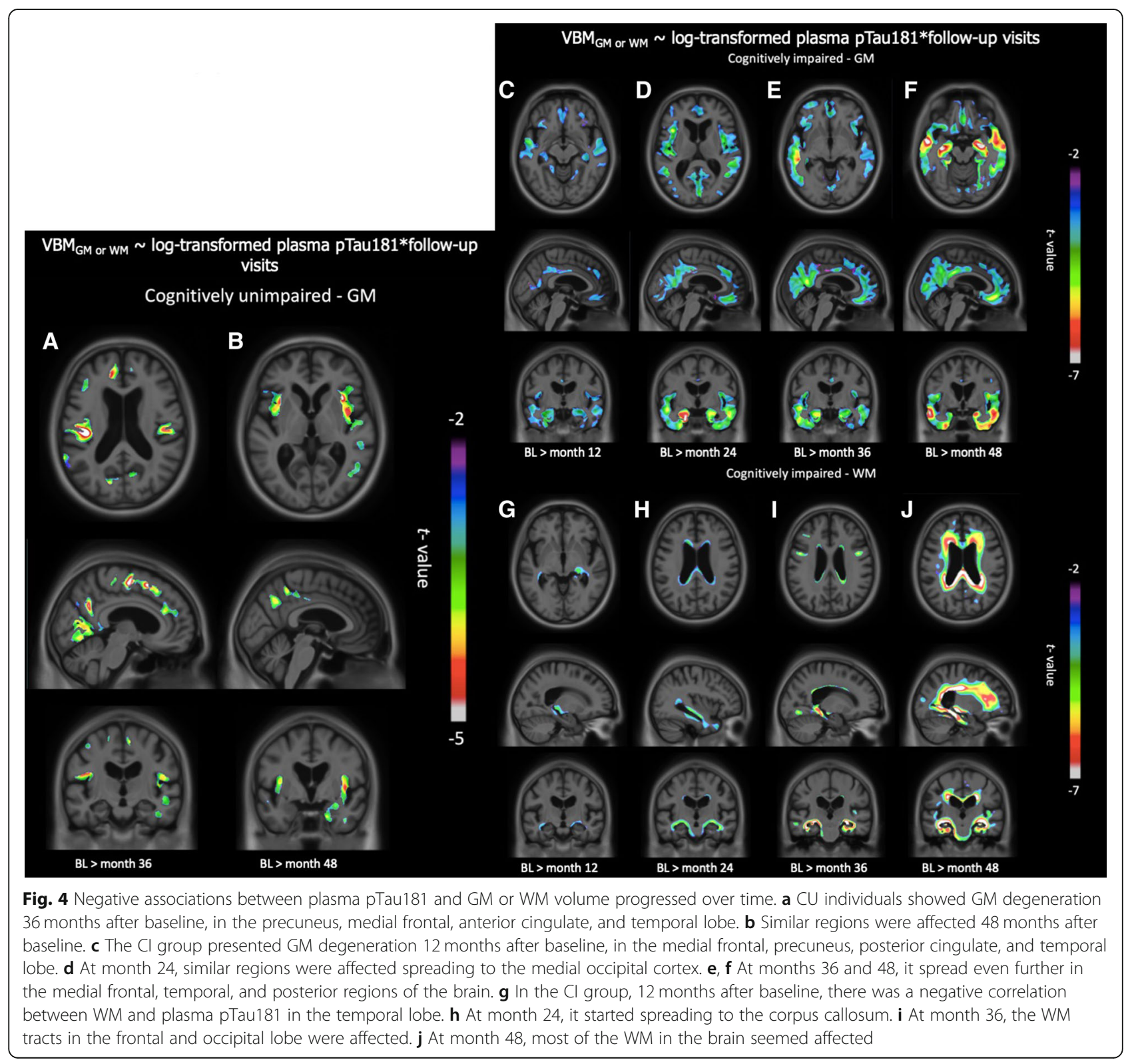




\section{Discussion}

In summary, we found that plasma pTau181 was associated with GM loss in both $\mathrm{CU}$ and $\mathrm{CI}$ groups, while its associations with WM loss were observed only in CI cross-sectionally. In CU individuals, plasma pTau181 predicted GM degeneration in $\mathrm{AD}$-related regions starting 36 months after baseline. In CI, plasma pTau181 predicted an incremental degeneration, in both GM and WM, which started in the typical AD-related brain regions, and encompassing the cortex and WM globally 4 years after the first assessment. The VBM changes seen as early as 12 months after baseline support the hypothesis that plasma pTau181 predicts imminent neurodegeneration.

Cross-sectional analyses suggested that measures of tau phosphorylation with plasma pTau181 inform about GM degeneration among CU individuals. The crosssectional associations occurred only in areas well known to be affected early in the AD process, such as the cingulate cortex, in which early amyloid deposition is often observed [14]. In CU, the anterior cingulate degeneration has been linked to complex attentional deficits and is known to be impaired along the clinical spectrum of AD [15]. Other studies also presented anterior cingulate atrophy as a predictor of conversion to dementia due to $\mathrm{AD}$ in memory-impaired individuals [16], suggesting the region is affected before the onset of cognitive symptoms.

In CI individuals, we observed a strong negative correlation between GM loss and plasma pTau181 in regions commonly affected in AD [17], such as the medial temporal lobe, the precuneus, and the anterior cingulate. The medial temporal region, which encompasses the hippocampus, is well known to be related to early atrophy in $\mathrm{AD}[18,19]$. The associations found between plasma pTau 181 and precuneus atrophy also corroborates the finding that this region is often impaired at the early stages of AD $[17,20]$. Similarly, as in the CU individuals, the CI group presented GM degeneration in the anterior cingulate, giving further support to the idea that neurodegeneration in this specific region is associated with tau hyperphosphorylation. Furthermore, we observed associations between plasma pTau181 and WM damage along the temporal lobe as well as the corpus callosum. Temporal WM atrophy has been consistently linked to aging and early AD [21]. The region is known to connect a network of memory-related areas. The associations between temporal WM and plasma pTau 181 might indicate a vulnerability of these WM tracts to hyperphosphorylation. Finally, the periventricular WM was also shown to correlate with plasma pTau181 in the CI group. Ventricular dilation and periventricular WM fibers have been presented as a biomarker of neurodegeneration [22]. Taken together, the cross-sectional analyses performed in this study provide evidence that high plasma pTau181 indicates neurodegeneration in brain regions vulnerable to early AD pathology.

Our longitudinal analyses revealed that plasma pTau181 predicted GM degeneration in various brain regions known to be affected in AD [17]. Particularly, the temporal lobe and posterior cingulate have shown atrophy in people with high plasma pTau181 baseline levels in $\mathrm{CU}$ individuals. Atrophy and tau deposition in both regions are well correlated with deficits of memory formation and retrieval in $\mathrm{AD}$ [23]. Functional alterations of the cingulate cortex imposed by $\mathrm{AD}$ pathophysiology have been proved to forecast dementia 2 years later [24]. Interestingly, we showed that plasma pTau181 predicts neurodegeneration in those specific regions, making it a possible earlier predictor of upcoming brain atrophy and possibly cognitive changes. By contrast, we did not observe associations between plasma pTau181 and longitudinal WM degeneration in cognitively unimpaired individuals. It is possible that WM is affected later in the disease process as compared to GM. Nevertheless, in CI individuals, we observed a strong negative correlation between plasma pTau181 and both GM and WM degeneration longitudinally. Plasma pTau181 predicted subsequent medial temporal, as well as precuneus, medial frontal, and medial occipital degeneration. Those brain regions are known to be included in the Default Mode Network, crucial for cognitive tasks [25]. AD pathophysiologies in these brain regions are also associated with memory dysfunction [26] and are known to accumulate neurofibrillary tangles [17]. It is well described that there is a decrease in the Default Mode Network connectivity along the continuum of normal aging to dementia [26]. The default mode alterations are associated with deficits in memory retrieval and envisioning the future, among others [25]. Regarding WM, plasma pTau181 predicted degeneration more specifically in the temporal and periventricular WM. WM abnormalities in these regions are considered crucial for memory formation and retrieval [21] and are also seen as markers of neurodegeneration in $\mathrm{AD}$ [22]. In WM, tau hyperphosphorylation can potentially come from glia or merely be associated to GM tau [27]. These results indicate that high plasma pTau181 in symptomatic cases harbingers AD-related neurodegeneration.

Further longitudinal analyses conducted at each FU MRI revealed how plasma pTau181 predicted neurodegeneration in both $\mathrm{CU}$ and $\mathrm{CI}$ groups. Although no effects were observed in the WM of CU individuals, plasma pTau181 predicted cortical neurodegeneration at month 36 after baseline in the temporal GM, the medial frontal cortex, the anterior cingulate, and the precuneus. These regions are known to be vulnerable to early $\mathrm{AD}$ pathology and important for memory [16-18]. The same 
analyses with time as an ordinal variable were conducted in the CI group; we observed an incremental degeneration of both WM and GM, resembling Braak staging [17]. At 12-month FU, we observed that plasma pTau181 was related to GM degeneration in the medial temporal, medial frontal, and precuneus regions. In the course of the 4 years post-baseline, we observed that the GM degeneration increased and spread more broadly to the entire cortex. At month 48, the medial temporal cortex, medial frontal cortex, and precuneus were significantly associated with plasma pTau181 concentrations. Similarly, the WM deterioration progresses with time. At month 12, alterations were restricted to the temporal lobe, affected early in $\mathrm{AD}$, while at month 48 , the frontal WM and the corpus callosum were heavily impaired. In the CI group, plasma pTau181 was able to predict imminent brain atrophy, which broadened incrementally to affect the entire brain. Taken together, these results suggest that plasma pTau181, in addition to being a cost-effective and scalable marker of future tau hyperphosphorylation, incorporates information regarding present and upcoming neurodegeneration.

The current findings between plasma pTau181 and brain atrophy corroborate previous research on CSF and plasma pTau181 [6, 28]. Higher levels of phosphorylated tau predicted neurodegeneration in the medial temporal and periventricular WM among other regions in aging and $\mathrm{AD}[28,29]$. In CI individuals, plasma pTau181 was correlated with degeneration of GM in the precuneus and temporal lobes [6]. It also predicted atrophy in the hippocampal region along the AD spectrum [1]. Bloodbased biomarkers, particularly pTau181, or hyperphosphorylated tau, represent an important step in facilitating disease diagnosis and possibly patient management. Moreover, blood-based biomarkers could serve to enrich a population for clinical trial selection. The last decade has focused on the research of biomarkers useful in $\mathrm{AD}$ [30], leading to the development of the current assay to assess plasma pTau181. The present study provides evidence of an added value associated with the new immunoassay for plasma pTau181. The higher the plasma pTau181 levels, the higher is the probability of coexisting neurodegeneration and the higher the likelihood of developing brain degeneration in the subsequent years. Indeed, among the $\mathrm{CU}$ individuals, it predicted cortical atrophy in AD-related brain regions 3 years later, while in the $\mathrm{CI}$ individuals, it forecasted imminent atrophy of both GM and WM as soon as 12 months later, with a progressive degeneration spreading all over the brain.

Other plasma markers have been studied in $A D$, such as neurofilament light (NfL) and amyloid- $\beta(\mathrm{A} \beta)$, which are also promising candidates for blood-based biomarkers [31]; however, both have shown some disadvantages. Indeed, NfL is a non-specific biomarker, as it has been related to other neurodegenerative conditions [32, 33] and is related to aging. Cerebral amyloidosis can be found in CU individuals [34] as well as a proportion of non-AD dementias. Furthermore, there is a significant production of peripheral $\mathrm{A} \beta$ expression [35] which may not be related to the $\mathrm{AD}$ process. Nevertheless, the current assay for plasma pTau181 is highly specific for AD [1] with the advantage to inform about coexistent and future neurodegeneration.

\section{Limitations}

The main strength of the study is to provide insights regarding levels of plasma pTau181, using a novel assessing method, and regional brain atrophy in a large cohort of well-characterized individuals. The assay was previously shown to be AD specific, when compared to other neurodegenerative conditions [1]. However, further research is required in larger cohorts with different tauopathies. There are various methodological limitations in the study, such as the fact that not all individuals had follow-up assessments every 12 months along the 4-year period post-baseline. Demographic differences were also present among groups, which were however accounted for in the statistical models. In ADNI [36], a multi-center cohort, and in other single-study cohorts [1], plasma pTau181 levels have been shown to increase with disease severity. Individuals presenting amyloid positivity had significantly higher levels of plasma pTau181 as compared to CU individuals [36]. In our analyses, the $\mathrm{CU}$ and $\mathrm{CI}$ groups present a significant difference in plasma pTau181 concentrations, albeit a certain overlap. It is thought to be due to the combination of $\mathrm{MCI}$ and $\mathrm{AD}$ together in the $\mathrm{CI}$ group, and the nonstratification depending on the amyloid status. Apart from these limitations, the new plasma pTau181 immunoassay could be proposed as a simple and scalable way to diagnose $\mathrm{AD}$ as it is highly associated with amyloidosis and neurofibrillary tangles and, according to our analysis, predict future brain atrophy in aging and dementia due to $\mathrm{AD}$. To conclude, we provide evidence that this novel test for plasma pTau181 has a strong negative correlation with brain atrophy patterns typical of $\mathrm{AD}$ and can also help predict subsequent neurodegeneration in aging and dementia due to AD.

\section{Conclusion}

The current study showed, in a large cohort of wellcharacterized individuals, that plasma pTau181 correlates with neurodegeneration at baseline and predicts further brain atrophy. Indeed, CU individuals mainly present GM atrophy, while CI individuals display both GM and WM damage. The incremental patterns of neurodegeneration involve brain regions related to $\mathrm{AD}$ 
pathologies. PET and CSF assessments being more expensive and not readily available, plasma pTau181 may then be a useful, cost-effective, and scalable biomarker to predict $\mathrm{AD}$-related neurodegeneration, with high specificity for $\mathrm{AD}$.

\section{Abbreviations}

AD: Alzheimer's disease; ADNI: Alzheimer's Disease Neuroimaging Initiative; $A \beta$ : Amyloid- $\beta$; CDR: Clinical Dementia Rating; Cl: Cognitively impaired; CSF: Cerebrospinal fluid; CU: Cognitively unimpaired; FU: Follow-up; GM: Gray matter; LM: Linear models; LME: Linear mixed effects; MCl: Mild cognitive impairment; MRI: Magnetic resonance imaging; NfL: Neurofilament light; PET: Positron emission tomography; pTau: Phosphorylated tau; RFT: Random field theory; VBM: Voxel-based morphometry; WM: White matter

\section{Supplementary Information}

Supplementary information accompanies this paper at https://doi.org/10. 1186/s13195-021-00802-X.

Additional file 1: Supplementary Figure 1. Correlations maps (Rmaps) of cross-sectional analyses, in both $\mathrm{CU}$ and $\mathrm{Cl}$ groups. Supplementary Figure 2. Longitudinal changes of plasma pTau181 in each individual. Supplementary Figure 3. Longitudinal plasma pTau181 changes among cognitively unimpaired and cognitively impaired individuals, stratified by $A \beta$ status.

\section{Acknowledgements}

CT would like to acknowledge the help received from Ugo Angles in computer coding.

Data used in the preparation of this article were obtained from the Alzheimer's Disease Neuroimaging Initiative (ADNI) database

(adni.loni.usc.edu). As such, the investigators within the ADNI contributed to the design and implementation of ADNI and/or provided data but did not participate in the analysis or writing of this report. A complete listing of ADNI investigators can be found at http://adni.loni.usc.edu/wp-content/ uploads/how_to_apply/ADNI_Acknowledgement_List.pdf.

\section{Authors' contributions}

$C T$ : Conception and design of the study, acquisition and analysis of the data, drafting a significant portion of the manuscript, figures. ALB: Conception and design of the study, acquisition and analysis of the data. JT: Conception and design of the study, acquisition and analysis of the data. TAP: Conception and design of the study, acquisition and analysis of the data. FL: Conception and design of the study, acquisition and analysis of the data. PSC: Acquisition and analysis of the data. MC: Conception and design of the study. SSM: Acquisition and analysis of the data. GB: Acquisition and analysis of the data. YTW: Acquisition and analysis of the data. JFA: Acquisition and analysis of the data. JLR: Acquisition and analysis of the data. AS: Acquisition and analysis of the data. NJA: Acquisition and analysis of the data. TKK: Acquisition and analysis of the data. KB: Conception and design of the study. $\mathrm{HZ}$ : Conception and design of the study. EDVS: Conception and design of the study. PH: Conception and design of the study. SG: Conception and design of the study. PRN: Conception and design of the study, drafting a significant portion of the manuscript or figures. The author(s) read and approved the final manuscript.

\section{Funding}

This work was supported by the Canadian Institutes of Health Research (CIHR) [MOP-11-51-31; RFN 152985, 159815, 162303], Canadian Consortium of Neurodegeneration and Aging (CCNA; MOP-11-51-31 -team 1), Weston Brain Institute, the Alzheimer's Association [NIRG-12-92090, NIRP-12-259245], and the Fonds de Recherche du Québec - Santé (FRQS; Chercheur Boursier, 2020-VICO-279314). TAP, P.R-N, and SG are members of the CIHR-CCNA Canadian Consortium of Neurodegeneration in Aging.

$\mathrm{HZ}$ is a Wallenberg Scholar supported by grants from the Swedish Research Council (\#2018-02532); the European Research Council (\#681712); the Swedish State Support for Clinical Research (\#ALFGBG-720931); the Alzheimer Drug Discovery Foundation (ADDF), USA (\#201809-2016862); and the UK
Dementia Research Institute at UCL. KB is supported by the Swedish Research Council (\#2017-00915); the Alzheimer Drug Discovery Foundation (ADDF), USA (\#RDAPB-201809-2016615); the Swedish Alzheimer Foundation (\#AF-742881); Hiärnfonden, Sweden (\#FO2017-0243); the Swedish state under the agreement between the Swedish government and the County Councils, the ALF-agreement (\#ALFGBG-715986); and European Union Joint Program for Neurodegenerative Disorders (JPND2019-466-236).

Data collection and sharing for this project was funded by the Alzheimer's Disease Neuroimaging Initiative (ADNI) (National Institutes of Health Grant U01 AG024904) and DOD ADNI (Department of Defense award number W81XWH-12-2-0012). ADNI is funded by the National Institute on Aging, the National Institute of Biomedical Imaging and Bioengineering, and through generous contributions from the following: AbbVie, Alzheimer's Association; Alzheimer's Drug Discovery Foundation; Araclon Biotech; BioClinica, Inc;; Biogen; Bristol-Myers Squibb Company; CereSpir, Inc.; Cogstate; Eisai Inc.; Elan Pharmaceuticals, Inc.; Eli Lilly and Company; Eurolmmun; F. Hoffmann-La Roche Ltd. and its affiliated company Genentech, Inc.; Fujirebio; GE Healthcare; IXICO Ltd.; Janssen Alzheimer Immunotherapy Research \& Development, LLC.; Johnson \& Johnson Pharmaceutical Research \& Development LLC.; Lumosity; Lundbeck; Merck \& Co., Inc.; Meso Scale Diagnostics, LLC.; NeuroRx Research; Neurotrack Technologies; Novartis Pharmaceuticals Corporation; Pfizer Inc.; Piramal Imaging; Servier; Takeda Pharmaceutical Company; and Transition Therapeutics. The Canadian Institutes of Health Research is providing funds to support ADNI clinical sites in Canada. Private sector contributions are facilitated by the Foundation for the National Institutes of Health (www.fnih.org). The grantee organization is the Northern California Institute for Research and Education, and the study is coordinated by the Alzheimer's Therapeutic Research Institute at the University of Southern California. ADNI data are disseminated by the Laboratory for Neuro Imaging at the University of Southern California.

Availability of data and materials

All data can be found on adni.loni.usc.edu.

\section{Declarations}

Ethics approval and consent to participate

Each ADNI site received approval from their Ethics Board to conduct the study. Written consent was obtained from all the research participants.

\section{Consent for publication}

Not applicable.

\section{Competing interests}

CT, ALB, JT, TAP, FL, PSC, MC, MS, SM, GB, YTW, JFA, NJA, TKK, EDVS, PH, SG, and PR-N have no disclosures to report.

$\mathrm{HZ}$ has served at scientific advisory boards for Denali, Roche Diagnostics, Wave, Samumed, Siemens Healthineers, Pinteon Therapeutics, and CogRx; has given lectures in symposia sponsored by Fujirebio, Alzecure, and Biogen; and is a co-founder of Brain Biomarker Solutions in Gothenburg AB (BBS), which is a part of the GU Ventures Incubator Program (outside submitted work). KB has served as a consultant, at advisory boards, or at data monitoring committees for Abcam, Axon, Biogen, Julius Clinical, Lilly, MagQu, Novartis, Roche Diagnostics, and Siemens Healthineers, and is a co-founder of Brain Biomarker Solutions in Gothenburg AB (BBS), which is a part of the GU Ventures Incubator Program (outside submitted work).

\section{Author details}

${ }^{1}$ The McGill University Research Centre for Studies in Aging, Douglas Hospital, McGill University, 875 La Salle Blvd - FBC room 3149, Montreal, QC H4H 1R3, Canada. ${ }^{2}$ Translational Neuroimaging Laboratory-McGill University, Montreal, QC, Canada. ${ }^{3}$ Douglas Hospital Research Centre, Verdun, QC, Canada. ${ }^{4}$ Department of Epidemiology and Biostatistics, McGill University, Montreal, QC, Canada. ${ }^{5}$ Department of Psychiatry and Neurochemistry, Institute of Neuroscience and Physiology, The Sahlgrenska Academy, University of Gothenburg, Gothenburg, Sweden. 'Wallenberg Centre for Molecular and Translational Medicine, University of Gothenburg, Gothenburg, Sweden. 'King's College London, Institute of Psychiatry, Psychology \& Neuroscience, Maurice Wohl Clinical Neuroscience Institute, London, UK. ${ }^{8} \mathrm{NIHR}$ Biomedical Research Centre for Mental Health \& Biomedical Research Unit for Dementia at South London \& Maudsley NHS Foundation, London, 
UK. ${ }^{9}$ Clinical Neurochemistry Laboratory, Sahlgrenska University Hospital, Mölndal, Sweden. ${ }^{10}$ UK Dementia Research Institute at UCL, London, UK. ${ }^{11}$ Department of Neurodegenerative Disease, UCL Institute of Neurology, London, UK. ${ }^{12}$ Center for Research on Brain, Language and Music, McGill university, Montreal, QC, Canada. ${ }^{13}$ Neurodegenerative disease groups, Montreal Neurological Institute, Montreal, QC, Canada. ${ }^{14}$ Le Centre intégré universitaire de santé et de services sociaux (CIUSSS) de I'Ouest-de-I'̂le-de-Montréal, Montreal, QC, Canada. ${ }^{15}$ Department of Neurology and Neurosurgery, Psychiatry and Pharmacology and Therapeutics, McGill University, Montreal, Canada.

\section{Received: 23 October 2020 Accepted: 8 March 2021} Published online: 29 March 2021

\section{References}

1. Karikari TK, Pascoal TA, Ashton NJ, Janelidze S, Benedet AL, Rodriguez JL, Chamoun M, Savard M, Kang MS, Therriault J, Schöll M, Massarweh G, Soucy JP, Höglund K, Brinkmalm G, Mattsson N, Palmqvist S, Gauthier S, Stomrud E, Zetterberg H, Hansson O, Rosa-Neto P, Blennow K. Blood phosphorylated tau 181 as a biomarker for Alzheimer's disease: a diagnostic performance and prediction modelling study using data from four prospective cohorts. Lancet Neurol. 2020;19(5):422-33. https:// doi.org/10.1016/S1474-4422(20)30071-5.

2. Mielke MM, Hagen $C E, X u J$, Chai X, Vemuri P, Lowe VJ, Airey DC, Knopman DS, Roberts RO, Machulda MM, Jack CR Jr, Petersen RC, Dage JL. Plasma phospho-tau181 increases with Alzheimer's disease clinical severity and is associated with tau- and amyloid-positron emission tomography. Alzheimers Dement. 2018;14(8):989-97. https://doi.org/10.1 016/j.jalz.2018.02.013.

3. Tatebe H, Kasai T, Ohmichi T, Kishi Y, Kakeya T, Waragai M, et al. Quantification of plasma phosphorylated tau to use as a biomarker for brain Alzheimer pathology: pilot case-control studies including patients with Alzheimer's disease and Down syndrome. Mol Neurodegener. 2017;12(1):1-11.

4. Yang S-Y, Chiu M-J, Chen T-F, Lin C-H, Chen W-P, Yang C-C. P3-255: assay of plasma phosphorylated tau protein (threonine 181) and total tau protein in vascular dementia, Parkinson's disease, frontotemporal dementia, and earlystage Alzheimer's disease. Alzheimer's Dement. 2018;14(7S_Part_22):P1172.

5. Janelidze S, Mattsson N, Palmqvist S, Smith R, Beach TG, Serrano GE, Chai X, Proctor NK, Eichenlaub U, Zetterberg H, Blennow K, Reiman EM, Stomrud E, Dage $J$, Hansson O. Plasma P-tau181 in Alzheimer's disease: relationship to other biomarkers, differential diagnosis, neuropathology and longitudinal progression to Alzheimer's dementia. Nat Med. 2020;26(3):379-86. https:// doi.org/10.1038/s41591-020-0755-1.

6. Thijssen EH, La Joie R, Wolf A, Strom A, Wang P, laccarino L, et al. Diagnostic value of plasma phosphorylated tau181 in Alzheimer's disease and frontotemporal lobar degeneration. Nat Med. 2020;26(3):387-97. https://doi. org/10.1038/s41591-020-0762-2

7. Benussi A, Karikari TK, Ashton NJ, Gazzina S, Premi E, Benussi L, et al. Diagnostic and prognostic value of serum NfL and p-Tau 181 in frontotemporal lobar degeneration. J Neurol Neurosurg Psychiatry. 2020;91(9):960-7.

8. McKhann G, Drachman D, Folstein M, Katzman R, Price D, Stadlan EM. Clinical diagnosis of Alzheimer's disease: report of the NINCDS-ADRDA Work Group* under the auspices of Department of Health and Human Services Task Force on Alzheimer's disease. Neurology. 1984;34(7):939-44. https://doi. org/10.1212/WNL.34.7.939.

9. Jack CR, Bennett DA, Blennow K, Carrillo MC, Dunn B, Haeberlein SB, et al. NIA-AA research framework: toward a biological definition of Alzheimer's disease. Alzheimers Dement. 2018;14(4):535-62. https://doi.org/10.1016/j.ja Iz.2018.02.018.

10. Jack CR, Bernstein MA, Fox NC, Thompson P, Alexander G, Harvey D, Borowski B, Britson PJ, L. Whitwell J, Ward C, Dale AM, Felmlee JP, Gunter JL, Hill DLG, Killiany R, Schuff N, Fox-Bosetti S, Lin C, Studholme C, DeCarli CS, Gunnar Krueger, Ward HA, Metzger GJ, Scott KT, Mallozzi R, Blezek D, Levy J, Debbins JP, Fleisher AS, Albert M, Green R, Bartzokis G, Glover G, Mugler J, Weiner MW, ADNI Study. The Alzheimer's Disease Neuroimaging Initiative (ADNI): MRI methods. J Magn Reson Imaging. 2008;27(4):685-91. https://doi. org/10.1002/jmri.21049.

11. Ashburner J. A fast diffeomorphic image registration algorithm. Neuroimage. 2007;38(1):95-113. https://doi.org/10.1016/j.neuroimage.2007. 07.007 .
12. Mathotaarachchi S, Wang S, Shin M, Pascoal TA, Benedet AL, Kang MS, et al. VoxelStats: a MATLAB package for multi-modal voxel-wise brain image analysis. Front Neuroinform. 2016;10:1-12.

13. Worsley KJ, Taylor JE, Tomaiuolo F, Lerch J. Unified univariate and multivariate random field theory. Neuroimage. 2004;23(SUPPL. 1):189-95.

14. Palmqvist $S$, Schöll M, Strandberg O, Mattsson N, Stomrud E, Zetterberg H, et al. Earliest accumulation of $\beta$-amyloid occurs within the default-mode network and concurrently affects brain connectivity. Nat Commun. 2017;8(1) https://doi.org/10.1038/s41467-017-01150-x.

15. Mesulam MM, Nobre AC, Kim YH, Parrish TB, Gitelman DR. Heterogeneity of cingulate contributions to spatial attention. Neuroimage. 2001;13(6):106572. https://doi.org/10.1006/nimg.2001.0768.

16. Killiany RJ, Gomez-Isla T, Moss M, Kikinis R, Sandor T, Jolesz F, Tanzi R, Jones K, Hyman BT, Albert MS. Use of structural magnetic resonance imaging to predict who will get Alzheimer's disease. Ann Neurol. 2000;47(4):430-9. https://doi.org/10.1002/1531-8249(200004)47:4<430::AID-ANA5>3.0.CO;2-I.

17. Braak H, Braak E. Neuropathological stageing of Alzheimer-related changes. Acta Neuropathol. 1991;82(4):239-59. https://doi.org/10.1007/BF00308809.

18. Frisoni GB, Testa C, Zorzan A, Sabattoli F, Beltramello A, Soininen H, Laakso MP. Detection of grey matter loss in mild Alzheimer's disease with voxel based morphometry. J Neurol Neurosurg Psychiatry. 2002;73(6):657-64. https://doi.org/10.1136/jnnp.73.6.657.

19. Ohnishi T, Matsuda H, Asada T, Tabira T, Uno M. Changes in brain morphology in Alzheimer disease and normal aging: is Alzheimer disease an exaggerated aging process? Am J Neuroradiol. 2001;22(9):1680-5.

20. Bailly M, Destrieux C, Hommet C, Mondon K, Cottier JP, Beaufils E, et al. Precuneus and cingulate cortex atrophy and hypometabolism in patients with Alzheimer's disease and mild cognitive impairment: MRI and 18F-FDG PET quantitative analysis using FreeSurfer. Biomed Res Int. 2015;2015(Mci). https://doi.org/10.1155/2015/583931.

21. Salat DH, Greve DN, Pacheco JL, Quinn BT, Helmer KG, Buckner RL, et al. Regional white matter volume differences in nondemented aging and Alzheimer's disease. Neuroimage. 2009;44(4):1247-58. https://doi.org/10.101 6/j.neuroimage.2008.10.030.

22. Teipel SJ, Bayer W, Alexander GE, Zebuhr Y, Teichberg D, Kulic L, Schapiro MB, Möller HJ, Rapoport SI, Hampel H. Progression of corpus callosum atrophy in Alzheimer disease. Arch Neurol. 2002;59(2):243-8. https://doi. org/10.1001/archneur.59.2.243.

23. Zhou Y, Dougherty JH, Hubner KF, Bai B, Cannon RL, Hutson RK. Abnormal connectivity in the posterior cingulate and hippocampus in early Alzheimer's disease and mild cognitive impairment. Alzheimers Dement. 2008;4(4):265-70. https://doi.org/10.1016/j.jalz.2008.04.006.

24. Huang C, Wahlund L-O, Svensson L, Winblad B, Julin P. Cingulate cortex hypoperfusion predicts Alzheimer's disease in mild cognitive impairment. BMC Neurol. 2002;12:2-9.

25. Buckner RL, Andrews-Hanna JR, Schacter DL. The brain's default network: anatomy, function, and relevance to disease. Ann N Y Acad Sci. 2008; 1124(1):1-38. https://doi.org/10.1196/annals.1440.011.

26. Hafkemeijer A, van der Grond J, Rombouts SARB. Imaging the default mode network in aging and dementia. Biochim Biophys Acta - Mol Basis Dis. 2012; 1822(3):431-41. https://doi.org/10.1016/j.bbadis.2011.07.008.

27. Kovacs GG, Ferrer I, Grinberg LT, Alafuzoff I, Attems J, Budka H, Cairns NJ, Crary JF, Duyckaerts C, Ghetti B, Halliday GM, Ironside JW, Love S, Mackenzie IR, Munoz DG, Murray ME, Nelson PT, Takahashi H, Trojanowski JQ, Ansorge O, Arzberger T, Baborie A, Beach TG, Bieniek KF, Bigio EH, Bodi I, Dugger BN, Feany M, Gelpi E, Gentleman SM, Giaccone G, Hatanpaa KJ, Heale R, Hof PR, Hofer M, Hortobágyi T, Jellinger K, Jicha GA, Ince P, Kofler J, Kövari E, Kril J,, Mann DM, Matej R, McKee AC, McLean C, Milenkovic I, Montine TJ, Murayama S, Lee EB, Rahimi J, Rodriguez RD, Rozemüller A, Schneider JA, Schultz C, Seeley W, Seilhean D, Smith C, Tagliavini F, Takao M, Thal DR, Toledo JB, Tolnay M, Troncoso JC, Vinters HV, Weis S, Wharton SB, White CL III, Wisniewski T, Woulfe JM, Yamada M, Dickson DW. Aging-related tau astrogliopathy (ARTAG): harmonized evaluation strategy. Acta Neuropathol. 2016;131(1):87-102. https://doi.org/10.1007/s00401-015-1509-x.

28. Llibre-Guerra JJ, Li Y, Schindler SE, Gordon BA, Fagan AM, Morris JC, Benzinger TLS, Hassenstab J, Wang G, Allegri R, Berman SB, Chhatwal J, Farlow MR, Holtzman DM, Jucker M, Levin J, Noble JM, Salloway S, Schofield P, Karch C, Fox NC, Xiong C, Bateman RJ, McDade E. Association of longitudinal changes in cerebrospinal fluid total tau and phosphorylated tau 181 and brain atrophy with disease progression in patients with Alzheimer disease. JAMA Netw Open. 2019;2(12):e1917126. https://doi.org/10.1001/jamanetworkopen.2019.17126. 
29. Gispert JD, Monté GC, Falcon C, Tucholka A, Rojas S, Sánchez-Valle R, et al. CSF YKL-40 and pTau181 are related to different cerebral morphometric patterns in early AD. Neurobiol Aging. 2016;38:47-55. https://doi.org/10.101 6/j.neurobiolaging.2015.10.022.

30. O'Bryant SE, Mielke MM, Rissman RA, Lista S, Vanderstichele H, Zetterberg H, et al. Blood-based biomarkers in Alzheimer disease: current state of the science and a novel collaborative paradigm for advancing from discovery to clinic. Alzheimers Dement. 2017;13(1):45-58. https://doi.org/10.1016/j.jalz.201 6.09.014.

31. Benedet AL, Ashton NJ, Pascoal TA, Leuzy A, Mathotaarachchi S, Kang MS, et al. Plasma neurofilament light associates with Alzheimer's disease metabolic decline in amyloid-positive individuals. Alzheimer's Dement Diagn Assess Dis Monit. 2019;11:679-89.

32. De Marchis GM, Katan M, Barro C, Fladt J, Traenka C, Seiffge DJ, et al. Serum neurofilament light chain in patients with acute cerebrovascular events. Eur J Neurol. 2018;25(3):562-8. https://doi.org/10.1111/ene.13554.

33. Hansson O, Janelidze S, Hall S, Magdalinou N, Lees AJ, Andreasson U, Norgren N, Linder J, Forsgren L, Constantinescu R, Zetterberg H, Blennow K, For the Swedish BioFINDER study. Blood-based NfL: a biomarker for differential diagnosis of parkinsonian disorder. Neurol Int. 2017;88(10):930-7. Available from: http://www.ncbi.nlm.nih.gov/pmc/articles/PMC5333515/\%0A http://www.nelft.nhs.uk/library\%0Ahttp://www.neurology.org/lookup/doi/1 0.1212 WNL.0000000000003680

34. Chételat G, La Joie R, Villain N, Perrotin A, De La Sayette V, Eustache F, et al. Amyloid imaging in cognitively normal individuals, at-risk populations and preclinical Alzheimer's disease. Neurolmage Clin. 2013;2(1):356-65. https:// doi.org/10.1016/j.nicl.2013.02.006.

35. Citron M, Vigo-Pelfrey C, Teplow DB, Miller C, Schenk D, Johnston J, Winblad B, Venizelos N, Lannfelt L, Selkoe DJ. Excessive production of amyloid $\beta$ protein by peripheral cells of symptomatic and presymptomatic patients carrying the Swedish familial Alzheimer disease mutation. Proc Natl Acad Sci U S A. 1994;91(25):11993-7. https://doi.org/10.1073/pnas.91.25.11993.

36. Karikari TK, Benedet AL, Ashton NJ, Lantero Rodriquez J, Snellman A, SuárezCalvet $\mathrm{M}$, et al. Diagnostic performance and prediction of clinical progression of plasma phospho-tau181 in the Alzheimer's Disease Neuroimaging Initiative. Mol Psychiatry. 2020;26(2):429-42. https://doi.org/1 0.1038/s41380-020-00923-z

\section{Publisher's Note}

Springer Nature remains neutral with regard to jurisdictional claims in published maps and institutional affiliations.

Ready to submit your research? Choose BMC and benefit from:

- fast, convenient online submission

- thorough peer review by experienced researchers in your field

- rapid publication on acceptance

- support for research data, including large and complex data types

- gold Open Access which fosters wider collaboration and increased citations

- maximum visibility for your research: over $100 \mathrm{M}$ website views per year

At $\mathrm{BMC}$, research is always in progress.

Learn more biomedcentral.com/submissions 